\title{
The myths surrounding mild stimulation in vitro fertilization (IVF)
}

Raoul Orvieto ${ }^{1,2^{*}}$, Valeria Stella Vanni ${ }^{1,3}$ and Norbert Gleicher ${ }^{4,5,6,7}$

\begin{abstract}
So-called mild controlled ovarian hyperstimulation $(\mathrm{mCOH})$ has in recent years increased in popularity, claiming to be safer and more patient-friendly, while also improving in vitro fertilization (IVF) outcomes. We here challenge the International Society for Mild Approaches in Assisted Reproduction (ISMAAR) definition of mild stimulation, and especially address four fundamental issues, where our review found conventional $\mathrm{COH}(\mathrm{cCOH})$ advantageous over $\mathrm{mCOH}$. They are: prevalence of severe ovarian hyperstimulation syndrome (OHSS), oocyte/embryo quality, pregnancy/live birth rates, and cost. We conclude that an objective review of the literature does not support the routine utilization of $\mathrm{mCOH}$ in assisted reproduction.
\end{abstract}

Keywords: Ovarian stimulation, Aneuploidy, Cost-effectiveness, Ovarian hyperstimulation syndrome (OHSS), In vitro fertilization (IVF), Pregnancy rates, Live birth rates

\section{Background}

Controlled ovarian hyperstimulation $(\mathrm{COH})$ is essential for in vitro fertilization (IVF) success since it facilitates recruitment of multiple oocytes and, thereby, also often allows for multiple embryo transfers (ETs) [1], without causing ovarian hyperstimulation syndrome (OHSS). In recent years, milder approaches to $\mathrm{COH}$ have become increasingly popular, aiming to offer safer and more patientfriendly approaches to reduce patient stress, complications such as OHSS, and cost of IVF cycles [2, 3], while allegedly improving endometrial receptivity, embryo quality and implantation potential [4].

Since investigators used various different definitions and terminologies to describe mild controlled ovarian hyperstimulation $(\mathrm{mCOH})$, the Rotterdam-based International Society for Mild Approaches in Assisted Reproduction (ISMAAR) Consensus Group on Terminology for Ovarian Stimulation for IVF, in 2007, proposed a concise and simplified revised nomenclature for different approaches to ovarian stimulation for IVF [5].

The term natural or unstimulated cycle IVF was proposed when IVF is carried out during a spontaneous

\footnotetext{
* Correspondence: Raoul.orvieto@sheba.health.gov.il

${ }^{1}$ Infertility and IVF unit, Department of Obstetrics and Gynecology, Chaim

Sheba Medical Center, Tel Hashomer, Ramat Gan, Israel

${ }^{2}$ The Tarnesby-Tarnowski Chair for Family Planning and Fertility Regulation,

Sackler Faculty of Medicine, Tel-Aviv University, Tel-Aviv, Israel

Full list of author information is available at the end of the article
}

menstrual cycle without gonadotropin support, aiming to collect the naturally selected single oocyte of the cycle. $\mathrm{mCOH}$ was defined as follicle stimulating hormone (FSH) or human menopausal gonadotropin (HMG) administered at lower doses, and/or for a shorter duration in a GnRH antagonist co-treated cycle, or when oral compounds (anti-estrogens, or aromatase inhibitors) are used, either alone or in combination with gonadotropins, aiming for collection of 2-7 oocytes. cCOH was defined by use of GnRH analogues (long/flare agonist or antagonist protocols) with conventional doses of stimulation with FSH or HMG, aiming to collect eight or more oocytes.

These definitions can, however, be quite confusing. For example, offering $\mathrm{mCOH}$ with a daily gonadotropin dose $<150 \mathrm{IU}$, some patients may respond more vigorously than expected, yielding more than 8 oocytes. Such an oocyte count, however, is already in the $\mathrm{cCOH}$ range defined by ISMAAR. How, then, should such a cycle be defined, According to stimulation $(\mathrm{mCOH})$, or patient response $(\mathrm{cCOH})$ ?

Four other fundamental issues require review:

\section{Prevalence of severe OHSS}

Best IVF is characterized by balance between optimal ovarian stimulation with successful treatment outcomes and minimal rates of severe OHSS and/or (especially higher order) multiple pregnancies. Individualization of treatments, based on patient risk factors, cycle responses, 
elective single embryo transfer and the option of freezing all embryos have in susceptible cases the potential of reducing the risks and severity of the syndrome [6]. Withholding ovulation-inducing triggers of hCG, or replacing hCG with GnRH agonist (GnRHa) triggers may further reduce severe early OHSS. Especially GnRHa trigger, combining $\mathrm{GnRH}$ antagonist co-treatment and GnRHa trigger, has recently been increasinly utilized to eliminate severe early OHSS in support the OHSS-free clinic [7, 8].

Significantly reduced clinical pregnancies and increased first trimester pregnancy loss with such treatments $[9,10]$, however, popularized efforts to improve reproductive outcome through all-freeze policies, fresh transfers with intensive luteal support and fresh transfers with low-dose hCG supplementation. We previously published a comprehensive review of suggested strategies, to which the reader is referred [6]. It addresses the role of $\mathrm{GnRH}$ antagonists in $\mathrm{COH}$ protocols, the use of different luteal rescue protocols, the role of cabergoline and the ability to transfer embryos at blastocyst stage.

Utilizing this knowledge, severe OHSS is no longer a complication to fear, as we practically, have eliminated it from our practice for over a decade.

\section{Oocyte/embryo quality}

A study by Baart et al. [11] raised concerns after demonstrating lower embryo aneuploidy following $\mathrm{mCOH}$ compared to $\mathrm{cCOH}$. While $\mathrm{mCOH}$ produced significantly fewer oocytes and embryos, both regimens generated similar numbers of chromosomally normal embryos. Bart et al., therefore, concluded that differences in aneuploid embryos suggests that mitotic segregation errors may increase with growing gonadotropin dosages. On the contrary, several other studies have disputed the aforementioned observation, demonstrating a comparable aneuploidy rate in stimulated and un-stimulated cycles.

In 2012, Labarta et al. [12] compared unstimulated and stimulated IVF cycles in the same young ( $<35 \mathrm{yrs}$ ) egg donors. Using in those days preimplantation genetic screening (PGS) with fluorescence in situ hybridization (FISH) on cleavage-stage embryos, these authors reported similar aneuploidy rates in unstimulated and stimulated cycle (34.8\% and $40.6 \%$, respectively), with no in-between group differences in embryo quality and types of chromosomal abnormalities. These aneuploidy rates concurred with $36.4 \%$ rates already in 2009 reported by Verpoest et al. in unstimulated cycles [13]. Also in 2012, using similar early-stage PGS technologies, Gleicher et al. [14] in normally fertile women undergoing IVF for sex selection purposes, reported mild increases in aneuploidy with increasing gonadotropin dosages; but those increases were more than compensated for by larger numbers of transferrable euploid embryos in women who received higher gonadotropin stimulation because of larger oocyte yields. Already in1992, Gras et al. [15] reported that the rate of aneuploidy in nonstimulated oocytes was comparable to oocytes obtained after $\mathrm{COH}$ with either clomiphene/hMG or the flare GnRHa protocol.

Like Labarta et al. [12] and Gleicher et al. [14], Baart et al. [11] used FISH to determine copy number of only nine chromosome pairs of cleavage-stage embryos on day-3 after fertilization, a method since discarded due to high imprecision $[16,17]$. Aneuploidy rates reported by Baart et al. (66\% and 67\% percent, respectively) were also much higher than those by Labarta et al. [12] and Verpoest et al. [13] (34.8\% and 36.4\%, respectively). They also compared the aneuploidy rates, comparing presumed aneuploid and mosaic embryos when analyzing two blastomers. As we now know, mosaicism is much more common than previously appreciated and, likely, a normal physiological phenomenon of developing embryos with, still, strong innate abilities to self-correct downstream beyond blastocyst stage [reviewed in [17]]. This was best demonstrated by the achievement of surprisingly high normal live birth rates after transfer of mosaic/aneuploid embryos [18-20]. Adding, however, up rates of normal euploid and mosaic embryos in the study by Baart et al. [11], the percentages of clinically likely normal embryos $(28+33=61 \%$ v.s $39+21=60 \%)$, in $\mathrm{mCOH}$ and $\mathrm{cCOH}$ groups, suddenly, no longer differs.

A recent study [21] of patients undergoing IVF cycles involving $\mathrm{COH}$ and trophectoderm biopsy for PGS has demonstrated that the degree of exposure to exogenous gonadotropins did not significantly modify the likelihood of aneuploidy in patients with a normal ovarian response to stimulation (not requiring $\mathrm{COH}$ beyond cycle day 12). Patients requiring prolonged $\mathrm{COH}$ were demonstrated to have elevated odds of aneuploidy with increasing cumulative gonadotropin dose. Finding that may reflect an increased tendency towards oocyte and embryonic aneuploidy in patients with a diminished response to gonadotropin stimulation.

We, therefore, conclude that there is no reliable evidence to suggest that more aggressive ovarian stimulation increases aneuploidy rates in embryos to significant degrees.

\section{Pregnancy/live birth rates}

Several studies have dealt with pregnancyllive birth rates in $\mathrm{mCOH}$ vs $\mathrm{cCOH}$, demonstrating a huge heterogeneity in terms of inclusion criteria, numbers of oocyte retrieved/embryo transferred, or day of the transfer. Verberg et al. [22] conducted a meta-analysis, aiming to investigate whether retrieval of low numbers of oocytes following $\mathrm{mCOH}$ is associated with impaired implantation rates. Including three randomized controlled trials of a total of 592 first treatment cycles, optimal embryo implantation 
rates were observed with retrieved 5 oocytes following $\mathrm{mCOH}$ (31\%), versus 10 oocytes following $\mathrm{cCOH}$ (29\%). These finding suggest that modest numbers of oocytes following $\mathrm{mCOH}$ are not a reflection of poor ovarian response.

Distribution of retrieved oocytes was similar for both protocols (range 0-28), with median (25-75 percentile) of 6 [3-10] and 9 [6-12] following $\mathrm{mCOH}$ and $\mathrm{cCOH}$, respectively. Overlapping results made it also impossible, to categorized patients to $\mathrm{mCOH}$ or $\mathrm{cCOH}$, based on the definition of the Rotterdam ISMAAR Consensus group [5]. Moreover, when comparing ongoing pregnancy rates (PR) per fresh transfer, they were significantly higher in the cCOH vs. $\mathrm{mCOH}(29 \%$ vs $15 \%$; $p<0.001$, respectively).

Since $\mathrm{mCOH}$ cycles more frequently than $\mathrm{cCOH}$ cycles fail to reach embryo transfer, outcome analysis with reference cycle start ("intent to treat") would, likely, demonstrate even more impressive differences in favor of $\mathrm{cCOH}$.

More impressive difference was also found comparing stimulated to unstimulated cycles. Sunkara et al. [23] recently analyzed anonymous data obtained from the Human Fertilisation and Embryology Authority (HFEA), involving 584,835 stimulated IVF cycles and 6168 unstimulated IVF cycles. The overall live birth rates were $4.7 \%$ per cycle following unstimulated fresh IVF versus $22.5 \%$ following stimulated fresh IVF. In other words, 3.5 times as many unstimulated IVF cycles are required to achieve one live birth compared to stimulated IVF.

Above noted advantages of $\mathrm{CCOH}$ were demonstrated in first fresh-cycle transfers. Those advantages would also become even more obvious if additional frozen-thawed cycle were to be included. Moreover, optimal embryo implantation rates observed with 5 oocytes following $\mathrm{mCOH}$ [22] are really irrelevant because they fall far below the required oocyte yields for a live birth, reported to be 14-15 metaphase II oocytes, 10 day- 2 or day- 3 embryos or 5 blastocysts in $70 \%$ of patients [24, 25].

It was recently also demonstrated [26], that the cumulative live birth rate (LBR) following the transfer of all fresh and frozen-thawed embryos after a single ovarian stimulation, significantly increases with the number of oocytes retrieved. High responders ( $>15$ oocytes) demonstrated a significantly higher LBR not only versus poor (0-3 oocytes) and suboptimal [4-9] responders, but also versus women with normal [10-15] ovarian response. While suboptimal responders had a better outcome compared with poor ovarian responders, this group had a significantly lower cumulative LBR compared with normal ovarian responders [26].

\section{Cost}

Groen et al. [27] evaluated the cost-effectiveness of modified natural cycle (MNC) versus $\mathrm{CCOH}$. MNC was not cost-effective, as conventional $\mathrm{COH}$ dominated
MNC with a higher cumulative LBR and lower cost per patient. LBR per cycle was 3.8 higher in the conventional vs. MNC $\mathrm{COH}$ (23\% and $6 \%$, respectively), while the cost was 1.8 higher (2110 vs 1150 Euro. Extrapolating the data to $\mathrm{mCOH}$, which involves more medication (gonadotropins), and taking into consideration the total reproductive potential of each initiated IVF cycle (i.e. fresh plus subsequent frozen/thawed transfers) with reference point cycle start (i.e.," intent to treat") [25], $\mathrm{cCOH}$ would be advantageous in term of costeffectiveness per cumulative LBR.

\section{Conclusion}

$\mathrm{mCOH}$ has been proposed to provide safer and more patient-friendly IVF, with improving outcomes. Upon careful review, it offers none of these advantages. Regarding occurrence of severe OHSS, oocyte/embryo quality, pregnancy/live birth rates and cost, $\mathrm{cCOH}$ is at least comparable or sometime superior over $\mathrm{mCOH}$, discrediting the concept of using $\mathrm{mCOH}$ in routine IVF. Further large prospective studies are needed to compare and clarify the role of $\mathrm{mCOH}$ vs $\mathrm{cCOH}$ in the different subgroups of patients. Moreover, these studies may help fertility specialists in individualization and careful tailoring of the $\mathrm{COH}$ protocol for optimizing IVF success.

\section{Abbreviations \\ $\mathrm{cCOH}$ : Conventional controlled ovarian hyperstimulation; $\mathrm{COH}$ : Controlled ovarian hyperstimulation; ET: embryo transfers; FISH: fluorescence in situ hybridization; GnRHa: GnRH agonist; HFEA: Human Fertilisation and Embryology Authority; HMG: human menopausal gonadotropin; ISMAAR: International Society for Mild Approaches in Assisted Reproduction; IVF: in vitro fertilization; $\mathrm{mCOH}$ : mild controlled ovarian hyperstimulation; MNC: modified natural cycle; OHSS: ovarian hyperstimulation syndrome; PGS: preimplantation genetic screening}

\section{Acknowledgements}

The authors would like to thank the 'Memorial Fund Griffini Miglierina' within the 'Fondazione Comunitaria del Varesotto Onlus' for non-restricted financial support to Dr. VSV during the completion of the study.

Funding

This manuscript was not supported by specific funding.

\section{Authors' contributions}

All authors contributed to the concept of the manuscript; R.O. wrote the first draft of the manuscript. All authors, however, contributed to substantial changes of the manuscript in further drafts. The final draft before submission was approved by all authors.

\section{Competing interests}

RO is the journal' EIC. VSV and NG have nothing to declare.

\section{Consent for publication}

NA.

Ethics approval and consent to participate Not applicable (a review article).

\section{Publisher's Note}

Springer Nature remains neutral with regard to jurisdictional claims in published maps and institutional affiliations. 


\section{Author details}

'Infertility and IVF unit, Department of Obstetrics and Gynecology, Chaim Sheba Medical Center, Tel Hashomer, Ramat Gan, Israel. ${ }^{2}$ The Tarnesby-Tarnowski Chair for Family Planning and Fertility Regulation, Sackler Faculty of Medicine, Tel-Aviv University, Tel-Aviv, Israel. ${ }^{3}$ Centro Scienze Natalità, Department of Obstetrics and Gynecology, IRCCS Ospedale San Raffaele, Milano, Italy. ${ }^{4}$ The Center for Human Reproduction, New York, N.Y 10021, USA. ${ }^{5}$ Foundation for Reproductive Medicine, New York, N.Y 10022, USA. 'Laboratory of Stem Cell Biology and Molecular Embryology, The Rockefeller University, New York, N.Y 10065, USA. ${ }^{7}$ Department of Obstetrics and Gynecology, University of Vienna School of Medicine, 1090 Vienna, Austria.

Received: 21 April 2017 Accepted: 17 June 2017

Published online: 24 June 2017

\section{References}

1. Penzias AS. Improving results with assisted reproductive technologies: individualized patient-tailored strategies for ovulation induction. Reprod Biomed Online. 2004:9:43-6.

2. Pelinck MJ, Vogel NEA, Hoek A, Simons AHM, Arts EGJM, Mochtar MH, et al. Cumulative pregnancy rates after three cycles of minimal stimulation IVF and results according to subfertility diagnosis: a multicentre cohort study. Hum Reprod. 2006;21:2375-83.

3. Heijnen E, Marinus JC, De Klerk C, Polinder S, Beckers NGM, Klinkert ER, et al. A mild treatment strategy for in-vitro fertilisation: a randomised noninferiority trial. Lancet. 2007;369:743-9.

4. Verberg MFG, Macklon NS, Nargund G, Frydman R, Devroey P, Broekmans FJ, et al. Mild ovarian stimulation for IVF. Hum Reprod Update. 2009;15:13-29.

5. Nargund G, Fauser BCJM, Macklon NS, Ombelet W, Nygren K, Frydman R. For the Rotterdam ISMAAR Consensus Group on Terminology for Ovarian Stimulation for IVF. The ISMAAR proposal on terminology for ovarian stimulation for IVF. Hum Reprod. 2007;22:2801-4.

6. Orvieto R. Ovarian hyperstimulation syndrome- an optimal solution for an unresolved enigma. J Ov Res. 2013;6:77.

7. Orvieto R. Can we eliminate severe ovarian hyperstimulation syndrome? Hum Reprod. 2005:20:320-2.

8. Devroey P, Polyzos NP, Blockeel C. An OHSS-Free Clinic by segmentation of IVF treatment. Hum Reprod. 2011;6:2593-7.

9. Griesinger G, Diedrich K, Devroey P, Kolibianakis EM. GnRH agonist for triggering final oocyte maturation in the $\mathrm{GnRH}$ antagonist ovarian hyperstimulation protocol: a systematic review and meta-analysis. Hum Reprod Update. 2006;12:159-68.

10. Orvieto R, Rabinson J, Meltzer S, Zohav E, Anteby E, Homburg R. Substituting HCG with GnRH agonist to trigger final follicular maturation-a retrospective comparison of three different ovarian stimulation protocols. Reprod Biomed Online. 2006;13:198-201.

11. Baart EB, Martini E, Eijkemans MJ, Van Opstal D, Beckers NG, Verhoeff A, et al. Milder ovarian stimulation for in-vitro fertilization reduces aneuploidy in the human preimplantation embryo: a randomized controlled trial. Hum Reprod. 2007;22:980-8.

12. Labarta E, Bosch E, Alama P, Rubio C, Rodrigo L, Pellicer A. Moderate ovarian stimulation does not increase the incidence of human embryo chromosomal abnormalities in in vitro fertilization cycles. J Clin EndocrinolMetab. 2012;97:1987-94.

13. Verpoest W, Fauser BC, Papanikolaou E, Staessen C, Van Landuyt L, Donoso $P$, et al. Chromosomal aneuploidy in embryos conceived with unstimulated cycle IVF. Hum Reprod. 2008;23:2369-71.

14. Gleicher N, Kim A, Weghofer A, Barad DH. Lessons from elective in vitro fertilization (IVF) in, principally, non-infertile women. Reprod Biol Endocrinol. 2012;10:48.

15. Gras L, McBain J, Trounson A, Kola I. The incidence of chromosomal aneuploidy in stimulated and unstimulated (natural) uninseminated human oocytes. Hum Reprod. 1992;7:1396-401.

16. Mastenbroek S, Twisk M, van Echten-Arends J, Sikkema Raddatz B, Kovaar $J C$, Verhoeve $H R$, et al. In vitro fertilization with preimplantation genetic screening. N Engl J Med. 2007;357:359.

17. Gleicher N, Orvieto R. Is the hypothesis of preimplantation genetic screening (PGS) still supportable? A review. J Ovarian Res. 2017;10:21.

18. Gleicher N, Vidali A, Braverman J, Kushnir VA, Albertini DF, Barad DH. Further evidence against use of PGS in poor prognosis patients: report of normal births after transfer of embryos reported as aneuploid. Fertil Steril. 2015;104(Suppl 3):e9.
19. Gleicher N, Vidali A, Braverman J, Kushnir VA, Barad DH, Hudson C, Wu YG Wang Q, Zhang L, Albertini DF. Accuracy of preimplantation genetic screening (PGS) is compromised by degree of mosaicism of human embryos. Reprod Biol Endocrinol 2016b;14:54

20. Greco E, Minasi G, Fiorentino F. Healthy babies after intrauterine transfer of mosaic aneuploidy blastocysts. N Engl J Med. 2015;373:2089-90.

21. Sekhon L, Shaia K, Santistevan A, Cohn KH, Lee JA, Beim PY, Copperman AB. The cumulative dose of gonadotropins used for controlled ovarian stimulation does not influence the odds of embryonic aneuploidy in patients with normal ovarian response. J Assist Reprod Genet. 2017 Mar 20. doi:10.1007/s10815-017-0909-3. [Epub ahead of print]

22. Verberg MFG, Eijkemans MJC, Macklon NS, Heijnen EMEW, Baart EB, Hohmann FP, et al. The clinical significance of the retrieval of a low number of oocytes following mild ovarian stimulation for IVF: a meta-analysis. Hum Reprod Update. 2009;15:5-12.

23. Sunkara SK, La Marca A, Polyzos NP, Seed PT, Khalaf Y. Live birth and perinatal outcomes following stimulated and unstimulated IVF: analysis of over two decades of a nationwide data. Hum Reprod. 2016;31:2261-7.

24. Garrido N, Bellver J, Remoh J, Simon C, Pellicer A. Cumulative live-birth rates per total number of embryos needed to reach newborn in consecutive in vitro fertilization (IVF) cycles: a new approach to measuring the likelihood of IVF success. Fertil Steril. 2011:96:40-6.

25. Sunkara SK, Rittenberg V, Raine-Fenning N, Bhattacharya S, Zamora J, Coomarasamy A. Association between the number of eggs and live birth in IVF treatment: an analysis of 400135 treatment cycles. Hum Reprod. 2011;26:1768-74.

26. Drakopoulos P, Blockeel C, Stoop D, Camus M, de Vos M, Tournaye H, et al. Conventional ovarian stimulation and single embryo transfer for IVF/ICSI. How many oocytes do we need to maximize cumulative live birth rates after utilization of all fresh and frozen embryos? Hum Reprod. 2016;31:370-6.

27. Groen $\mathrm{H}$, Tonch $\mathrm{N}$, Simons AH, van der Veen F, Hoek A, Land JA. Modified natural cycle versus controlled ovarian hyperstimulation IVF: a costeffectiveness evaluation of three simulated treatment scenarios. Hum Reprod. 2013:28:3236-46.

\section{Submit your next manuscript to BioMed Central and we will help you at every step:}

- We accept pre-submission inquiries

- Our selector tool helps you to find the most relevant journal

- We provide round the clock customer support

- Convenient online submission

- Thorough peer review

- Inclusion in PubMed and all major indexing services

- Maximum visibility for your research

Submit your manuscript at www.biomedcentral.com/submit
Biomed Central 